\title{
Cavo-atrial thrombectomy prior to hepatectomy for hepatocellular carcinoma with tumor thrombus in the right atrium: a case report
}

\author{
Shun-ichi Ariizumi ${ }^{1}$, Chizuo Kikuchi ${ }^{2}$, Fumiaki Tokitou', Shingo Yamashita ${ }^{1}$, Yoshihito Kotera' ${ }^{1}$, Akiko Omori ${ }^{1}$, \\ Takaaki Kato ${ }^{1}$, Satoshi Nemoto ${ }^{1}$, Hiroshi Niinami ${ }^{2}$ and Masakazu Yamamoto ${ }^{1 *}$
}

\begin{abstract}
Background: Hepatocellular carcinoma (HCC) with tumor thrombus (TT) in the right atrium is a critical condition. The general consensus is to perform hepatectomy prior to cavo-atrial thrombectomy because of the risk of uncontrollable bleeding during the liver transection after heparinization. However, sudden cardiac arrest due to the ball-valve effect and pulmonary embolism have been reported in cases of $\Pi$. Cavo-atrial thrombectomy prior to hepatectomy for HCC with $\Pi$ in the right atrium was successfully performed to prevent sudden cardiac arrest and pulmonary embolism.

Case presentation: Tumor thrombectomy under cardiopulmonary bypass with heparin and electrical ventricular fibrillation prior to hepatectomy was successfully performed to prevent sudden cardiac arrest or pulmonary embolism in a 75-year-old woman with a huge HCC and $\Pi$ in the right atrium. After the neutralization of heparin, right hepatectomy with tumor thrombectomy in the inferior vena cava was performed. The total operation time was $9 \mathrm{~h}$, and the total blood loss was $8200 \mathrm{~mL}$. The patient's postoperative course was uneventful, and she was discharged 14 days after surgery. One year after surgery, she is alive with HCC recurrence in the lung.

Conclusions: Cavo-atrial thrombectomy prior to hepatectomy for HCC with TT in the right atrium can be performed safely to prevent sudden cardiac arrest and pulmonary embolism by collaboration of cardiovascular surgeons and gastroenterological surgeons.
\end{abstract}

Keywords: Hepatocellular carcinoma, Tumor thrombus, Right atrium, Thrombectomy

\section{Background}

Hepatocellular carcinoma (HCC) with tumor thrombus (TT) in the right atrium is a critical condition [1]. There is no standard treatment strategy, and surgery is challenging. The median survival is reported to be from 1 to 4 months, and it may be complicated by lung metastasis, pulmonary embolism, heart failure, and sudden cardiac death $[1,2]$. There are no effective nonsurgical treatments, and while long-term survivors have been reported after surgery, surgical mortality is reported to be

\footnotetext{
* Correspondence: yamamoto.masakazu@twmu.ac.jp

'Department of Surgery, Institute of Gastroenterology, Tokyo Women's

Medical University, Kawada 8-1, Shinjuku-ku, Tokyo 162-0054, Japan

Full list of author information is available at the end of the article
}

$15 \%$ [1-5]. The general consensus is to perform hepatectomy prior to cavo-atrial thrombectomy, because of the risk of uncontrollable bleeding during the subsequent transection of the liver after heparinization [6]. However, cavo-atrial thrombectomy prior to hepatectomy should be considered in cases with TT which fully enters the right atrium, reaches the tricuspid valve, and is of the pedunculated type, in order to prevent the ball-valve effect or pulmonary embolism [2].

\section{Case presentation}

The patient was a 75-year-old woman who presented with severe bilateral leg edema and epigastralgia. There was no past history of chronic hepatitis or blood 
transfusion. Admission laboratory tests revealed an elevated serum aspartate aminotransferase level $(48 \mathrm{U} / \mathrm{L})$, mildly decreased serum albumin $(3.6 \mathrm{~g} / \mathrm{dL})$, mildly decreased serum prothrombin time (74\%), mildly elevated D-dimer $(12.9 \mu \mathrm{g} / \mathrm{mL})$, and mildly elevated fibrin degradation product $(12.6 \mu \mathrm{g} / \mathrm{mL})$. The tumor marker AFP was slightly elevated to $585 \mathrm{ng} / \mathrm{mL}$. The Child-Pugh classification was $\mathrm{A}$, and the indocyanine green retention rate at $15 \mathrm{~min}$ was $12 \%$. CT scans showed a huge tumor, 21 $\mathrm{cm}$ in diameter, in the right liver and TT, $37 \mathrm{~mm}$ in diameter, in the right atrium (Figs. $1 \mathrm{a}, \mathrm{b}$ and $2 \mathrm{a}$ ). The TT was pedunculated, swung like a pendulum with the heartbeat, and reached the tricuspid valve on a transesophageal ultrasound video (Fig. 2b). The symptomatic patient had a risk of sudden death due to TT in the right atrium. Therefore, we explained the high surgical mortality rate to the patient and her family, and they decided to proceed with surgery.

At first, a partial sternotomy was made, and cardiopulmonary bypass $(\mathrm{CPB})$ via the aorta, superior vena cava, and right femoral vein and electrical ventricular fibrillation were performed after intravenous injection of 25,000 units of heparin sodium. Although the TT in the right atrium was very soft and fragile, thrombectomy from the right atrium and inferior vena cava (IVC) was successful (Fig. 3a-d). After the removal of TT, the IVC was clamped with a tourniquet above the diaphragm and the right atrium was sutured with 5-0 Prolene. After DC defibrillation was carried out, CPB was stopped, the tourniquet on the IVC was released, and $100 \mathrm{mg}$ protamine was administered for the neutralization of heparin. The open heart surgery time was $23 \mathrm{~min}$, and the $\mathrm{CPB}$ time was $42 \mathrm{~min}$. Next, a reverse $\mathrm{T}$ incision was made, and right hepatectomy by anterior approach and IVC tumor thrombectomy were performed. After ligation of the anterior and posterior Glissonean pedicles, the liver parenchyma was transected under the Pringle maneuver and IVC clamping below the liver.
After the IVC and right hepatic vein were confirmed by anterior approach, the IVC was opened and a residual TT in the IVC was removed under total hepatic vascular exclusion (THVE) (Fig. 4a-d). Finally, the huge tumor was removed with the diaphragm without pulmonary embolism. The total operation time was $9 \mathrm{~h}$, and the total blood loss was $8200 \mathrm{~mL}$. The tumor was of the massive type macroscopically (Fig. 5), and cancer cells showed moderately to poorly differentiated HCC with invasion to the portal vein, hepatic vein, and diaphragm. The patient's postoperative course was uneventful, and she was discharged 14 days after surgery. She is still alive 14 months after surgery with recurrence in the lung.

\section{Discussion}

The general consensus is to perform hepatectomy prior to cavo-atrial thrombectomy, because of the risk of uncontrollable bleeding during the subsequent transection of the liver after heparinization. However, in cases of a large TT which enters the right atrium, reaches the tricuspid valve, and is of the pedunculated type, sudden cardiac arrest due to the ball-valve effect and pulmonary embolism have been reported. Therefore, cavo-atrial thrombectomy prior to hepatectomy should be considered as one of the options for HCC with TT in the right atrium [1-6].

Tsang et al. reported a case of HCC with TT in the right atrium treated with combined cavo-atrial thrombectomy and hepatectomy [2]. According to their report, liver transection by the anterior approach was carried out before thrombectomy. However, hepatectomy was converted to thrombectomy because unstable hemodynamics and uncontrollable bleeding (blood loss was $30 \mathrm{~L}$ ) developed as a result of right ventricular inflow obstruction secondary to the ball-valve effect. In our present case, cavo-atrial thrombectomy prior to hepatectomy was performed to prevent the ball-valve effect or pulmonary embolism because the TT swung like a pendulum with the heartbeat
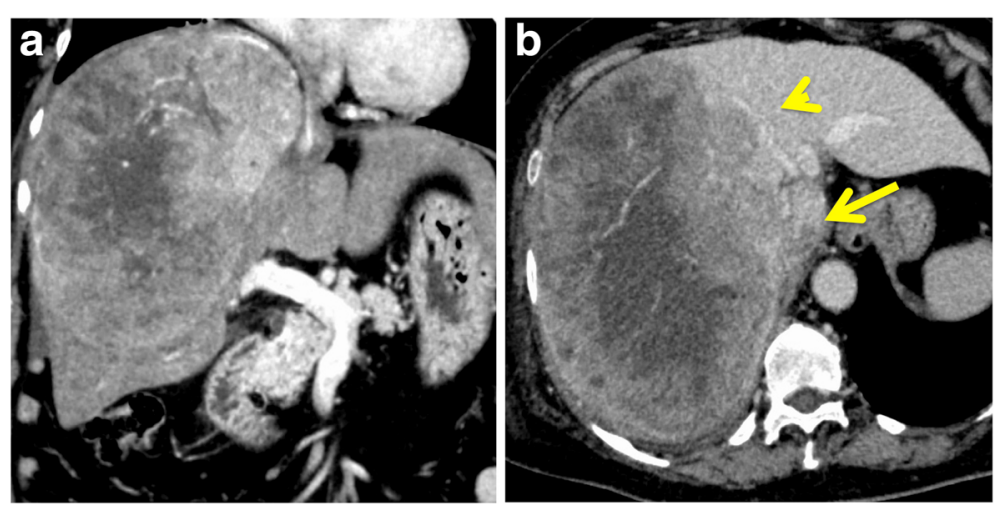

Fig. $1 \mathrm{CT}$ showed a huge tumor, $21 \mathrm{~cm}$ in diameter, in the right liver (a). The tumor compressed the middle hepatic vein (arrowhead) and inferior vena cava (arrow) (b) 


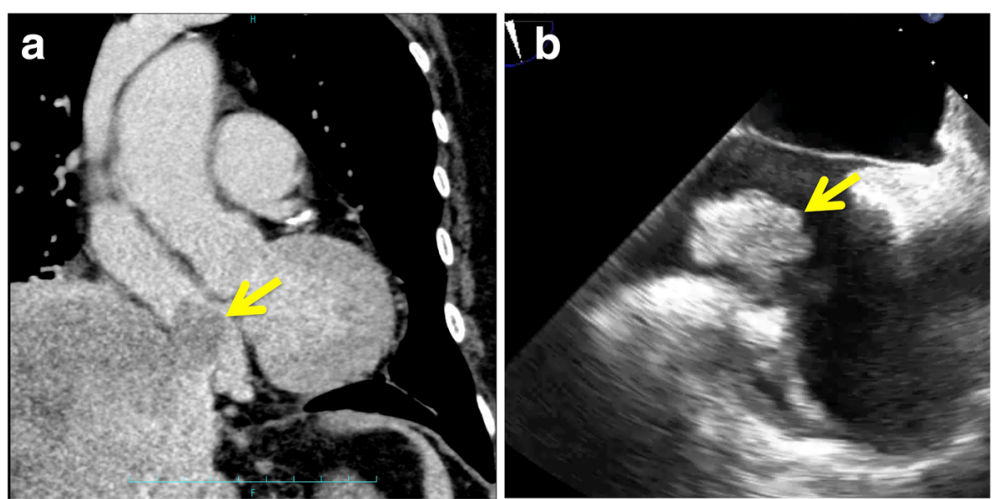

Fig. 2 CT showed a tumor thrombus in the right atrium (a, arrow). The tumor thrombus moved with the heartbeat and reached the tricuspid valve on transesophageal ultrasound (arrow) (b)

on a transesophageal ultrasound video. Transesophageal ultrasound video of a TT is useful for deciding to perform cavo-atrial thrombectomy prior to hepatectomy.

Sakamoto and Nagano classified TT in the IVC and/or right atrium into type I-III, and the intracardiac type (type III) requires hepatectomy plus thrombectomy under $\mathrm{CPB}$. There have been 14 previous reports of cases in which HCC with TT in the right atrium was successfully removed with hepatectomy prior to thrombectomy and CPB by collaboration of cardiovascular surgeons and gastroenterological surgeons (Table 1) [3, 7]. According to the reports, eight patients had no complications after surgery. However, one patient developed DIC, one patient had bleeding, and two patients had pleural effusion after surgery. Wakayama et al. reported six cases of $\mathrm{HCC}$ with TT in the right atrium treated with hepatectomy prior to thrombectomy with $\mathrm{CPB}$, two cases had controllable complications, but there was no surgical mortality [4]. At our institute, six patients with HCC and TT in the right atrium underwent hepatectomy prior to thrombectomy with or without CPB between 1986 and 2001.

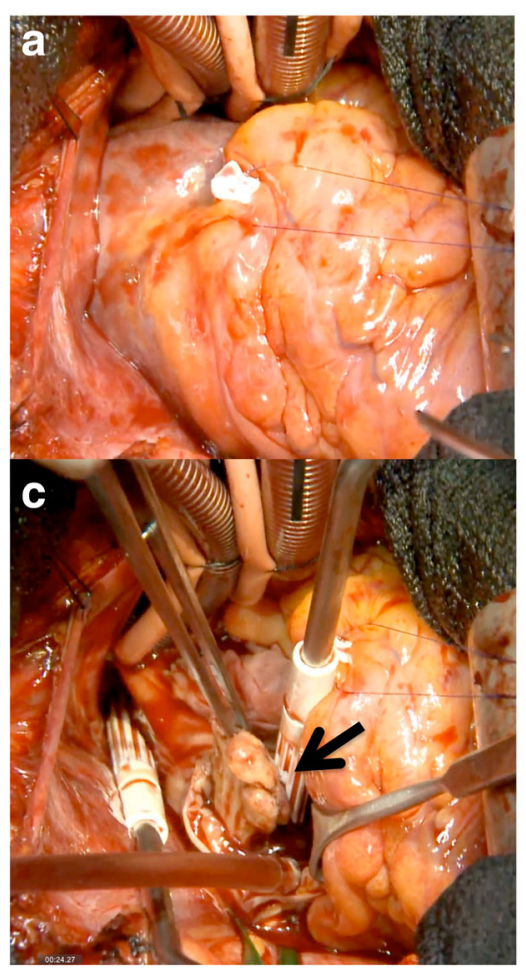

b

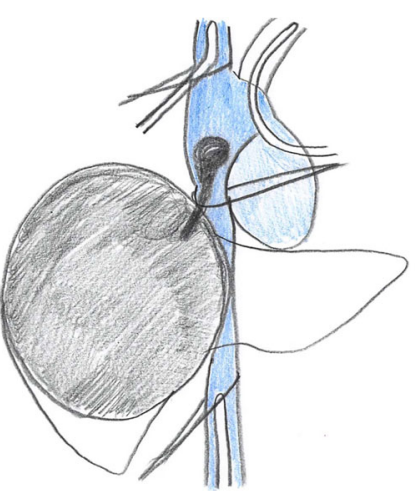

d

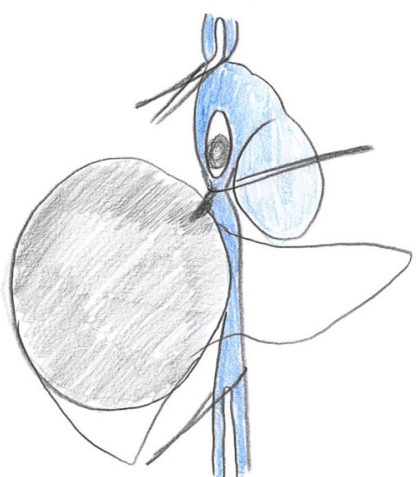

Fig. 3 Tumor thrombectomy in the right atrium under electrical ventricular fibrillation and cardiopulmonary bypass (CPB) was performed after intravenous injection of heparin sodium $(\mathbf{a}, \mathbf{b})$. The $\Pi$ in the right atrium was removed from the right atrium without pulmonary embolization $(\mathbf{c}, \mathbf{d})$. The open heart surgery time was $23 \mathrm{~min}$ 

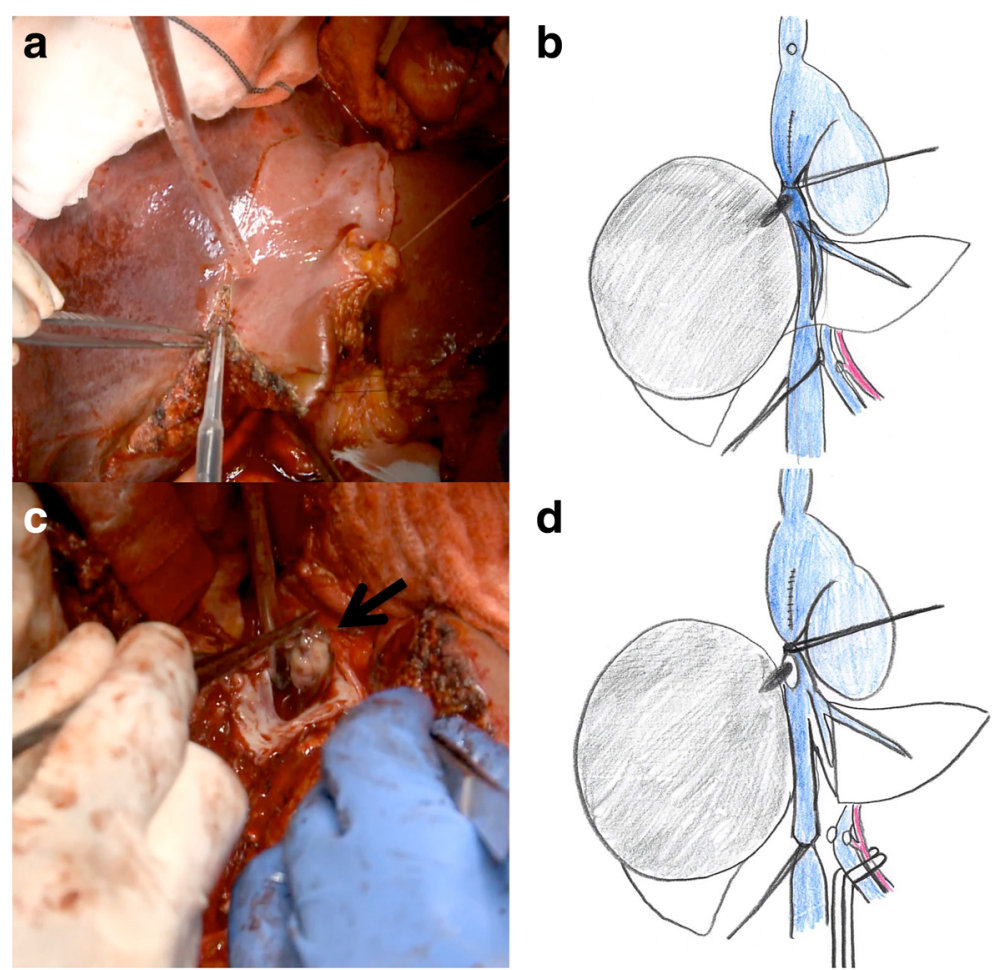

Fig. 4 Right hepatectomy by anterior approach $(\mathbf{a}, \mathbf{b})$ and IVC tumor thrombectomy were performed under total hepatic vascular exclusion after neutralization of heparin $(\mathbf{c}, \mathbf{d})$

Three of six patients died of sudden cardiac arrest during surgery. We therefore stopped surgery for HCC with atrial TT since 2001.

Recently, hepatectomy prior to thrombectomy without CPB has been attempted. There have been five previous reports of cases in which HCC with TT in the right atrium was successfully removed without CPB (Table 1) $[3,7]$. According to these reports, no complications or no mortality have been reported. When a TT is excluded from the right atrium after complete mobilization and caudal retraction of the liver, thrombectomy can be done with total hepatic vascular exclusion without $\mathrm{CPB}$. Hepatectomy prior to thrombectomy without $\mathrm{CPB}$ was performed in a 77-year-old man with HCC and TT in the right atrium at our institute in 2016. However, a pulmonary embolism due to TT developed in the left pulmonary artery. He died of multiple lung metastases 5 months after surgery. Therefore, hepatectomy prior to thrombectomy carries a risk of sudden cardiac arrest and pulmonary embolism during surgery.
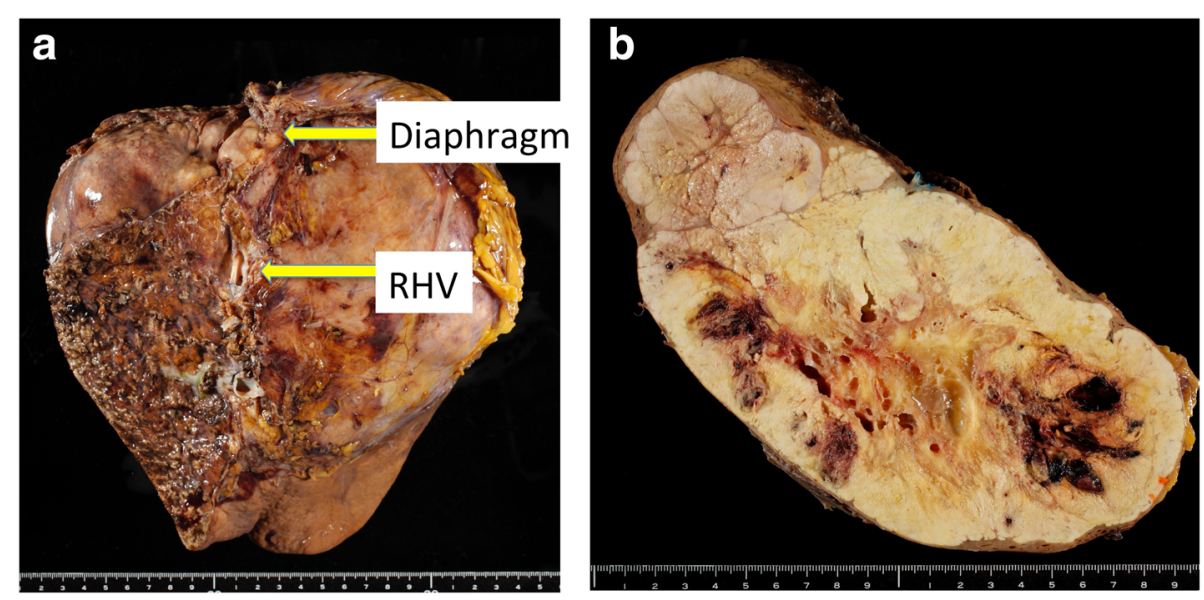

Fig. 5 The tumor showed a massive type macroscopically and HCC invaded to the diaphragm (a, b) 


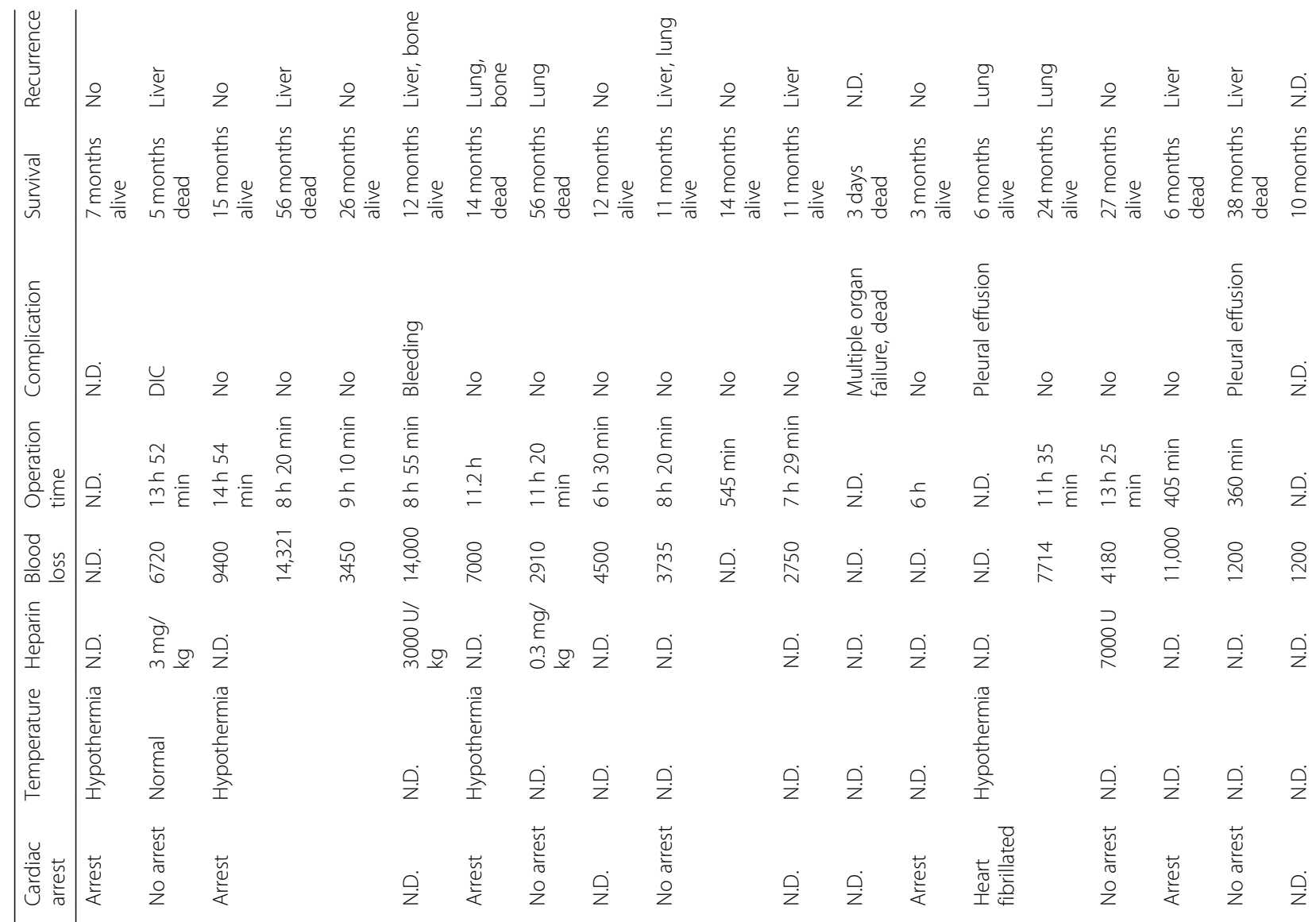

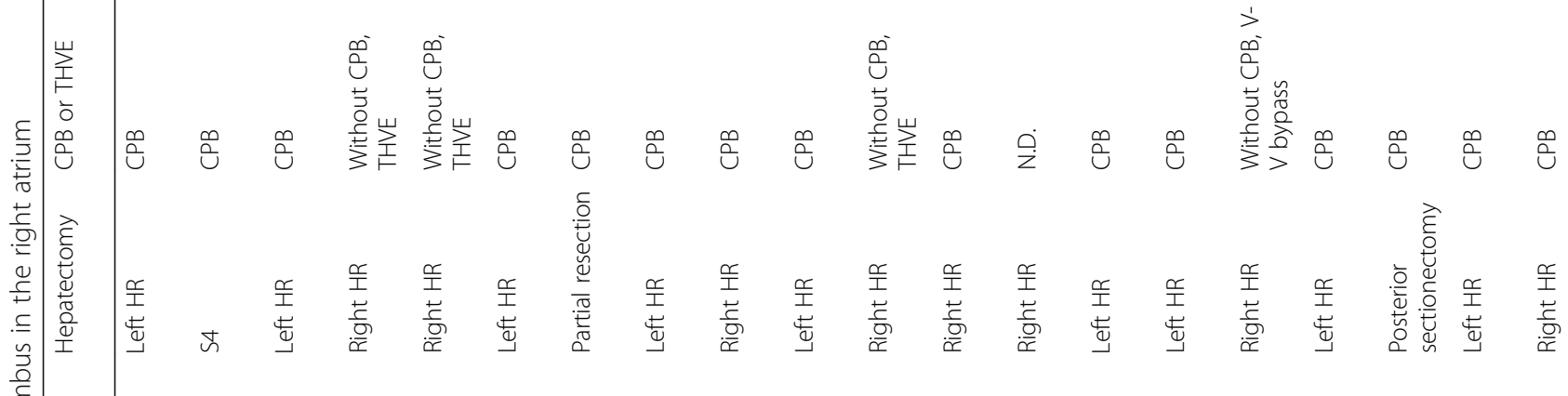

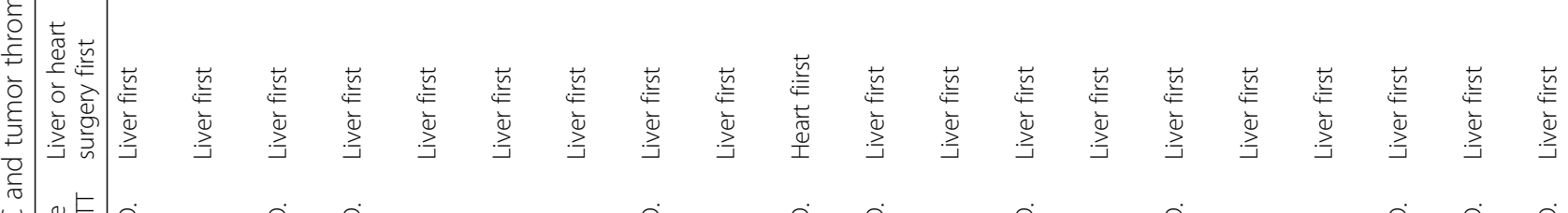

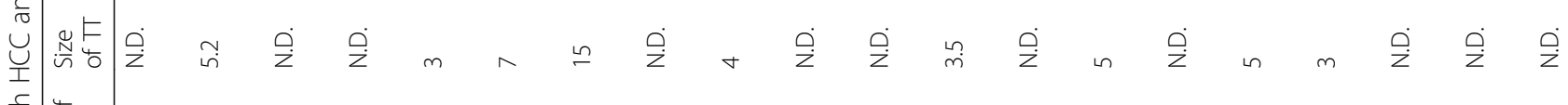
旁范

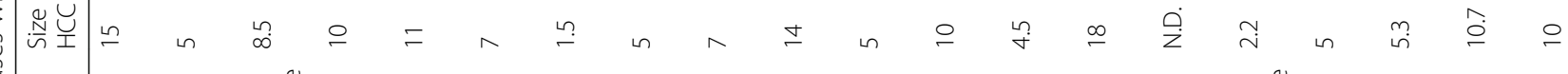

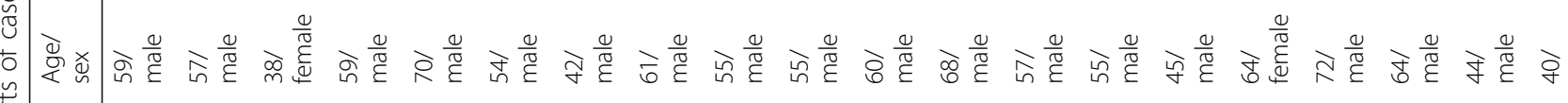
ণ্ঠ்

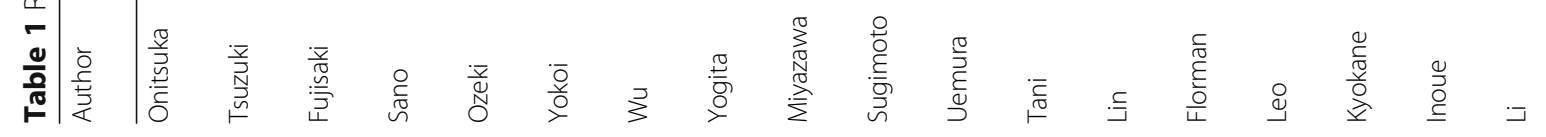




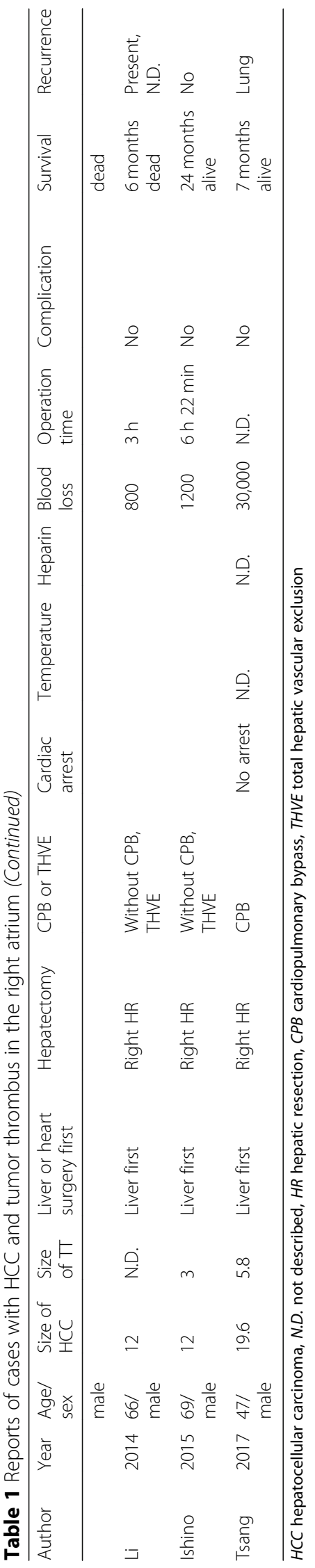


Sugimoto et al. first reported upfront cavo-atrial thrombectomy followed by hepatectomy to prevent sudden death or pulmonary embolism, and there have been no other reports to the best of our knowledge [8]. According to their Japanese report, thrombectomy prior to left hepatectomy was successfully performed without uncontrollable bleeding (blood loss was $3735 \mathrm{~mL}$ ). In our present case, most bleeding occurred during liver surgery (blood loss was $8200 \mathrm{~mL}$ ) due to heparinization. Thrombectomy prior to right hepatectomy will prevent uncontrollable bleeding due to inflow obstruction by the ball-valve effect.

For open heart surgery, heparin, cardiac arrest, hypothermia, and cardioplegia under $\mathrm{CPB}$ are required. Recently, cardiac surgery has been improved and open heart surgery with beating heart has become possible, removing any limitation of open heart surgery with beating heart. If the TT reaches the right ventricle, thrombectomy will be possible. For liver resection, major hepatectomy such as right or left hepatectomy was required because the size of $\mathrm{HCC}$ was huge and the TT also involved the right, middle, or left hepatic vein. In cases of HCC with TT in the right liver, the anterior approach to the IVC or RHV is recommended because this anterior approach is safe for huge HCC in the right liver.

In liver surgery for patients with HCC, safe and oncological management is important. Thrombectomy prior to hepatectomy requires cutting the TT in the IVC. This procedure may induce lung metastasis, and lung metastasis did occur in Sugimoto's and our patient. However, the rate of lung metastasis is also very high after hepatectomy prior to thrombectomy. Five of 22 cases in previous reports had lung metastasis after hepatectomy prior to thrombectomy. Wakayama et al reported that not only all six patients with TT in the right atrium but also six of seven patients with TT in the IVC had lung metastasis [4]. Furthermore, recent advanced molecular target therapy for lung metastasis is quite effective. Therefore, safe management is more important for patients with HCC and $\mathrm{TT}$ in the right atrium.

\section{Conclusions}

This is the first English-language report on cavo-atrial thrombectomy prior to hepatectomy. Cavo-atrial thrombectomy prior to hepatectomy for HCC with TT in the right atrium can be performed safely to prevent sudden cardiac arrest and pulmonary embolism. When a large TT is located in the right atrium and/or is of the pedunculated type, in which case there is a concern for sudden cardiac arrest and pulmonary embolism, cavo-atrial thrombectomy prior to hepatectomy is recommended.

\section{Abbreviations}

AFP: Alpha-fetoprotein; CT: Computed tomography; HCC: Hepatocellular carcinoma; IVC: Inferior vena cava; TT: Tumor thrombus

\section{Acknowledgements}

The authors are indebted to Associate Professor Raoul Breugelmans of the Department of English of Tokyo Medical University for his review of this manuscript.

\section{Funding}

None of the authors received any funding

\section{Availability of data and materials}

The authors declare that all the data in this article are available within the article.

\section{Authors' contributions}

All authors participated in a conference to plan the surgery and performed the surgery. All authors read and approved the final manuscript.

\section{Ethics approval and consent to participate}

Not applicable.

\section{Consent for publication}

The patient provided written informed consent to publish this case report and the accompanying images.

\section{Competing interests}

The authors declare that they have no competing interests.

\section{Publisher's Note}

Springer Nature remains neutral with regard to jurisdictional claims in published maps and institutional affiliations.

\section{Author details}

'Department of Surgery, Institute of Gastroenterology, Tokyo Women's Medical University, Kawada 8-1, Shinjuku-ku, Tokyo 162-0054, Japan.

${ }^{2}$ Department of Cardiovascular Surgery, Tokyo Women's Medical University, Tokyo, Japan.

Received: 18 January 2019 Accepted: 2 April 2019

Published online: 11 April 2019

\section{References}

1. Wang Y, Yuan L, Ge LI, Sun Y, Wei G. Survival benefit of surgical treatment for hepatocellular carcinoma with inferior vena cava/right atrium tumor thrombus: results of a retrospective cohort study. Ann Surg Oncol. 2013;20: 914-22.

2. Tsang J, Chan A, Chok K, Tsang F, Lo CM. Combined cavo-atrial thrombectomy and hepatectomy in hepatocellular carcinoma. Hepatobiliary Pancreat Dis Int. 2017;16:329-33.

3. Luo X, Zhang B, Dong S, Zhang B, Chen X. Hepatocellular carcinoma with tumor thrombus occupying the right atrium and portal vein. A case report and literature review. Medicine. 2015:94:1-5.

4. Wakayama K, Kamiyama T, Yokoo H, Kakisaka T, Kamachi H, Tsuruga Y, Nakanishi K, Shimamura T, Todo S, Taketomi A. Surgical management of hepatocellular carcinoma with tumor thrombi in the inferior vena cava or right atrium. World J Surg Oncol. 2013;11:259.

5. Pesi B, Giudici F, Moraldi L, Montesi G, Romagnoli S, Pinelli F, Stefano P, Batignani G. Hepatocellular carcinoma on cirrhosis complicated with tumor thrombi extended to the right atrium: results in three cases treated with major hepatectomy and thrombectomy under hypothermic cardiocirculatory arrest and literature review. World J Surg Oncol. 2016:14:83.

6. Sakamoto K, Nagano H. Outcomes of surgery for hepatocellular carcinoma with tumor thrombus in the inferior vena cava or right atrium. Surgery Today. 2018;48:819-24

7. Kyokane T, lyomasa S, Sawasaki N, et al. A case of hepatocellular carcinoma with intra-atrial tumor thrombus presenting pulmonary thromboemboli. Jpn J Gastroenterol Surg. 2010;43:398-404 In Japanese with English abstract.

8. Sugimoto $\mathrm{H}$, Inoue $\mathrm{S}$, Mori $\mathrm{T}$, et al. Tumor thrombus removal with hepatic resection using cardiopulmonary bypass in a patient with hepatocellular carcinoma extending into right atrium. Jpn J Gastroenterol Surg. 2004;37: 1737-42 In Japanese with English abstract. 